\title{
A Nonlinear Rank Regression Method for Ensemble Kalman Filter Data Assimilation
}

\author{
JEFFREY L. ANDERSON \\ Data Assimilation Research Section, National Center for Atmospheric Research, ${ }^{\mathrm{a}}$ Boulder, Colorado
}

(Manuscript received 27 December 2018, in final form 28 March 2019)

\begin{abstract}
It is possible to describe many variants of ensemble Kalman filters without loss of generality as the impact of a single observation on a single state variable. For most ensemble algorithms commonly applied to Earth system models, the computation of increments for the observation variable ensemble can be treated as a separate step from computing increments for the state variable ensemble. The state variable increments are normally computed from the observation increments by linear regression using the prior bivariate ensemble of the state and observation variable. Here, a new method that replaces the standard regression with a regression using the bivariate rank statistics is described. This rank regression is expected to be most effective when the relation between a state variable and an observation is nonlinear. The performance of standard versus rank regression is compared for both linear and nonlinear forward operators (also known as observation operators) using a low-order model. Rank regression in combination with a rank histogram filter in observation space produces better analyses than standard regression for cases with nonlinear forward operators and relatively large analysis error. Standard regression, in combination with either a rank histogram filter or an ensemble Kalman filter in observation space, produces the best results in other situations.
\end{abstract}

\section{Introduction}

Anderson (2003) shows that it is possible to describe many varieties of ensemble Kalman filters without loss of generality by simply describing the impact of a single observation on a single state variable. In particular, the classical perturbed observation ensemble Kalman filter (EnKF; Burgers et al. 1998; Houtekamer and Mitchell 1998), which is perhaps more accurately referred to as a stochastic EnKF (Vetra-Carvalho et al. 2018), the ensemble adjustment Kalman filter (EAKF; Anderson 2001), and other similar square root filters (Tippett et al. 2003) can be described in this way. The description can be simplified further by separating the computation of increments for the prior ensemble estimate of an observation from the computation of increments for a state variable given increments for the observation. For the standard EnKF and EAKF, the second step simply linearly regresses observation increments onto state variable increments using the prior bivariate ensemble of the observation and the state variable.

\footnotetext{
${ }^{a}$ The National Center for Atmospheric Research is sponsored by the National Science Foundation.
}

Corresponding author: Jeffrey L. Anderson, jla@ucar.edu
Subsequent studies have explored other methods for computing observation increments while continuing to use linear regression to compute state increments. For example, the rank histogram filter (RHF; Anderson 2010) is a nearly nonparametric method for computing observation increments. The prior ensemble is used to generate an approximate continuous prior distribution for the observation by partitioning the real line into bins containing equal probability mass, conceptually similar to the rank histogram (Anderson 1996; Hamill 2001).

The gamma/inverse gamma and inverse gamma/ gamma filters described in Bishop (2016) are other methods for computing observation increments. In the first case, the ensemble prior for an observation is approximated by a gamma distribution and observations with inverse gamma error distributions are assimilated; in the second case, the prior is inverse gamma and the observation error gamma. Again, standard linear regression is used to compute state variable increments given observation increments.

The quadratic filter of Hodyss (2012) and Hodyss et al. (2017) extends the ensemble Kalman filter by also estimating the third moment of the distributions. This requires defining auxiliary state estimation problems that assimilate pseudo-observations. Again, the increments 
for a state variable and pseudostate variables are computed using linear regression.

It is also possible to use algorithmic extensions in which a method other than linear regression is used to compute state variable increments from observation increments. For example, versions of the gamma class of filters (Posselt and Bishop 2018) lead to changes in the way state increments are computed from observation increments. Another example would be to select a particular form of nonlinear regression using the bivariate ensemble prior, for instance choosing a polynomial regression of a given order.

This paper describes another method for computing state variable increments from observation increments, the use of a regression based on the Spearman rank correlation instead of the Pearson correlation (least squares fit) used in standard regression. Section 2 describes low-order perfect model assimilation experiments that are used to compare results from standard and rank regression using simulated observations with uncorrelated observation errors. Section 3 describes the rank regression algorithm and includes illustrated comparisons to standard regression. Section 4 presents results comparing the capabilities of standard and rank regression in concert with the EAKF, EnKF, and RHF in observation space. Section 5 explores the computational cost of rank regression, and section 6 discusses the results and presents conclusions.

\section{Experimental design}

Perfect model data assimilation experiments are examined to compare capabilities of standard and rank regression. All experiments used the Manhattan release of the Data Assimilation Research Testbed (DART; Anderson et al. 2009). The Lorenz-96 model (L96; Lorenz and Emanuel 1998) is used with 40 variables, forcing $F=8.0$, and fourth-order Runge-Kutta for time differencing with a time step of 0.05 units. This is the same model used in previous data assimilation studies, including Anderson (2016) and many others such as Sakov et al. (2012). As in Anderson (2016), the state variables are assumed to be uniformly located on a cyclic $[0,1]$ domain with locations $0.025 i$, $i=1, \ldots, 40$.

The time mean of the ensemble mean posterior rootmean-square error is the metric used for comparing the performance of different assimilation algorithms. Unless noted, the term RMSE refers to this quantity:

$$
\mathrm{RMSE}=\sum_{t=1}^{5000}\left[\sum_{i=1}^{40}\left(\bar{x}_{i, t}^{u}-x_{i, t}^{T}\right)^{2} / 40\right] / 5000 .
$$

Here, $x$ is a state variable, the first subscript $i$ indexes the state variable (40 here), the second subscript $t$ indexes the time (all experiments described below will have 5000 assimilation times), superscript $T$ refers to the true state trajectory, superscript $u$ refers to the posterior (or updated) value, and an overbar is the ensemble mean.

Two different configurations of 40 "observation stations" are used. The "uniform" observation configuration observes at each of the 40 state variable locations. The "random" configuration observes at locations generated by 40 draws from a uniform $[0,1]$ distribution. When computing forward operators, the value of the state at each location is a linear interpolation between the two closest state variables. The 40 random station locations are the same as used in Anderson (2016). These locations can be computed using the random location generation procedure for the Lorenz-96 model in the default version of the DART Manhattan release (https:// www.image.ucar.edu/DAReS/DART/).

Let $x_{o}$ be the value of the state at an observation station. Four different forward operators are studied: identity $y_{o}=x_{o}$; square root $y_{o}=\operatorname{sgn}\left(x_{o}\right)\left(\left|x_{o}\right|\right)^{1 / 2}$; cube $y_{o}=x_{o}^{3}$; and square $y_{o}=x_{o}^{2}$.

Ten initial conditions for true model trajectories were generated by starting an L96 integration from a state of all zeroes except for a 1 for the first state variable. The model was advanced for 10 million steps, and the state was saved every 1 million steps.

Five truth integrations of L96 with different assimilation periods were initiated from each of the 10 initial conditions. Assimilation periods were 1, 2, 3, 6, and 12 model time steps, and each truth integration included 5500 observation times (e.g., the truth run with period $1 \mathrm{had}$ a total of 5500 time steps; the truth run for period 12 had a total of $12 \times 5500$ ).

For each of the four forward operators and for both uniform and random station configurations, several observation error variances were explored for each assimilation period; Table 1 lists the error variances. For the random station configuration, the smallest error variance with observations taken every time step for a given forward operator was selected so that the time mean prior RMSE for the best assimilations was approximately $10 \%$ of the climatological RMSE. The largest error variance for a given forward operator with assimilation every 12 model time steps has a prior RMSE that was approximately the same as the climatological RMSE.

A "case" is defined by the station configuration (uniform or random), the forward operator (identity, square root, cube, or square), the assimilation period $(1,2,3,6$, or 12 time steps), and the observation error variance (Table 1). Six different data assimilation "treatments" are applied to each case. The treatments 
TABLE 1. Observation error variances for assimilation cases. Ensembles of 80 members were used for cases in regular font, and ensembles of 20, 40, 80, and 160 members were used for cases in italics.

\begin{tabular}{lcl}
\hline \hline Forward operator & Uniform stations & Random stations \\
\hline Identity & 1 or 16 & $1,2,4$, or 16 \\
Square root & 0.5 or 2 & $0.25,0.5,1$, or 2 \\
Cube & 128 or 1024 & 128,1024, or 2048 \\
Square & & 16,64, or 256 \\
\hline
\end{tabular}

use standard regression or rank regression, with the observation increments being computed by the EAKF, the perturbed observation EnKF, or the non-Gaussian RHF with the default parameter settings from the DART Manhattan release. For example, one treatment uses rank regression with the RHF observation update.

Although adaptive inflation algorithms (Zhang et al. 2004; Anderson 2009; Whitaker and Hamill 2012; El Gharamti 2018) are effective, they make the complex problem of understanding the results even more complicated. To avoid this situation, empirically tuned fixed prior multiplicative inflation was used. Tuning of each case-treatment pair was done by trying 49 pairs of inflation and Gaspari-Cohn (1999) localization halfwidths; the values are listed in Table 2.

Data assimilation experiments began with each state variable of each ensemble being the true initial condition plus a draw from a unit normal. A total of 5500 data assimilation steps were done, and the first 500 steps were discarded. Five thousand steps were chosen for evaluation because it resulted in high statistical significance for detecting differences between the RMSE resulting from different treatments for selected cases (see discussion of paired $t$ tests below). Each case had 294 assimilations experiments, 49 inflation-localization pairs for each of the six treatments.

Initially, assimilation experiments had 80 ensemble members and were only done for the first of the 10 true trajectories. For each case, the assimilation experiment using standard regression that had the lowest RMSE was identified by its observation space increment method (EAKF, EnKF, RHF), localization and inflation. For example, the case with random station configuration and identity forward operator taken every step with error variance 3600 had smallest RMSE for an EnKF with no localization and inflation 1.02. The lowest RMSE experiment using rank regression was also identified for each case. These will be referred to as the best standard and rank treatments for a given case. For some experiments, the assimilation failed by either crashing or producing machine infinity results. Any experiment that failed was assigned infinite RMSE.
TABLE 2. Values of state space prior inflation and localization applied to each treatment for each case.

\begin{tabular}{lllllllc}
\hline \hline $\begin{array}{l}\text { Inflation } \\
\text { Localization } \\
\text { half-widths }\end{array}$ & 0.125 & 0.15 & 0.175 & 0.2 & 0.25 & 0.4 & No localization \\
\hline
\end{tabular}

To estimate the statistical significance of the differences between the best standard and rank regression treatments for a given case, assimilation experiments were run for the remaining nine true trajectories for both the best rank regression and standard regression treatments. The result was ten 5000-step assimilation experiments, each with different initial conditions, for the best rank and standard treatments for each case. Paired $t$ tests for the 10 pairs of RMSE were used to provide an estimate of the significance of the differences between the best rank regression and standard regression treatments.

The experiments were also carried out with ensemble sizes of 5,10,20, 40, and 160 members for all cases with random station locations and identity or square root forward operators. The same procedure as for 80 members was employed. The rank regression algorithm performed very poorly with 5 and 10 members, and those results are not discussed further.

\section{Rank regression for ensemble filters}

The regression step of a standard ensemble filter computes increments for a state variable ensemble given a prior ensemble estimate of an observation and corresponding observation increments. Let $\mathbf{x}$ be the prior state ensemble, $\mathbf{y}$ be the prior observation ensemble, $\Delta \mathbf{y}$ be the observation increments, and $\Delta \mathbf{x}$ be the state increments. A subscript $n$ refers to the individual ensemble members from 1 to the ensemble size $N$. The standard regression algorithm finds the slope $b$ of the least squares fit line for the state $\mathbf{x}$ as a function of the observation $\mathbf{y}$; that is, $b$ minimizes

$$
\sum_{n=1}^{N}\left[x_{n}-\left(a+b y_{n}\right)\right]^{2}
$$

Then the increments for $\mathbf{x}$ are $\Delta x_{n}=b \Delta y_{n}$.

The rank regression method assumes that there is a monotonic functional relation between the observation $y$ and the state $x$. The implications are discussed more in the next section. The algorithm proceeds as follows (note that $\tilde{r}$ refers to a generalized rank, a real value, whereas $r$ without a tilde refers to the traditional rank, which is an integer from 1 to $N$ ):

1) Compute the rank statistics $r^{y p}=\left(r_{1}^{y p}, r_{2}^{y p}, \ldots, r_{N}^{y p}\right)$ and $r^{x p}=\left(r_{1}^{x p}, r_{2}^{x p}, \ldots, r_{N}^{x p}\right)$ of the prior observation ensemble $\mathbf{y}^{p}$ and prior state ensemble $\mathbf{x}^{p}$. 
2) Compute the generalized rank of each member of the ensemble of posterior (updated) observations using the function $f$ described in the appendix: $\tilde{r}_{n}^{y u}=f\left(y_{n}+\Delta y_{n} ; y^{p}\right)$.

3) Compute the observation generalized rank increments for each member, $\Delta \tilde{r}_{n}^{y}=\tilde{r}_{n}^{y u}-r_{n}^{y p}$.

4) Compute the slope $b_{r}$ of the least squares fit line for the prior state rank statistics as a function of the prior observation rank statistics, that is, the value of $b_{r}$ that minimizes

$$
\sum_{n=1}^{N}\left[r_{n}^{x p}-\left(a_{r}+b_{r} r_{n}^{y p}\right)\right]^{2}
$$

5) Compute posterior state generalized rank statistics, $\tilde{r}_{n}^{x u}=r_{n}^{x p}+b_{r} \Delta \tilde{r}_{n}^{y}$.

6) Convert the ensemble of posterior state generalized rank statistics to posterior state values, $x_{n}^{u}=f^{-1}\left(\tilde{r}_{n}^{x u} ; x^{p}\right)$.

The subscript $n$ spans the ensemble members, $n=1, \ldots, N$.

Figure 1 displays the prior bivariate ensemble for an observation and a state variable along with posterior ensembles from standard and rank regression. These examples are from the case with uniform observation locations, a square root forward operator, assimilation period of 12 time steps, and observation error variance of 0.5 with the best inflation and localization for the standard regression. In Fig. 1a, the state variable and observation are at the same location so that the observation $y_{o}$ is related to the state variable $x$ by the signed square root, $y_{o}=\operatorname{sgn}(x)(|x|)^{1 / 2}$. The prior ensemble shows the monotonic functional relation between the observation and the state. The rank regression posterior ensemble has a distribution that is similar to a subsection of the prior ensemble. However, the standard regression posterior from the standard regression is quite different from any subset of the prior.

Figure $1 b$ shows a more nuanced result. In this case, the state variable is located two grid intervals away from the observation so there is no functional relationship between the observation and the state variable. Posterior ensembles from the standard and rank regression are different, but it is not obvious that one is superior to the other.

In the uniform observation configuration, only 1 in 40 of the regressions are for a collocated observation and state variable for which a functional relationship exists; in the random station configuration, none of the regressions have a functional relationship. It is unclear whether the advantages of the rank regression shown in Fig. 1a extend to situations like that in Fig. 1b. Of course, most real assimilation problems never have observations of a model state variable, so the uniform
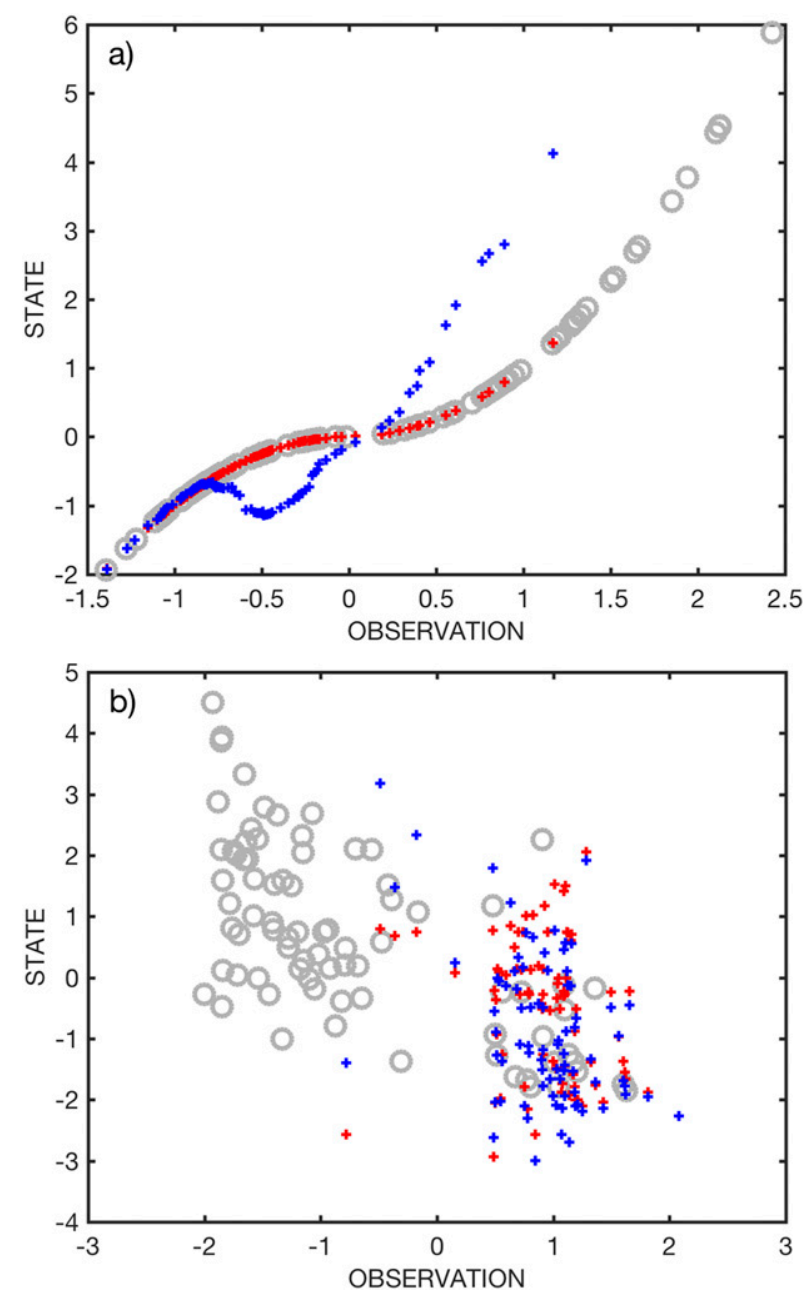

FIG. 1. Comparison of posterior ensemble from rank regression (red plus signs) and standard regression (blue plus signs) for updates of a state variable given the same prior ensemble (open gray circles) and observation increments. The examples are taken from the case with 40 uniformly located observation stations with observational error variance of 0.5 and assimilation period of 12 with a square root forward operator. Results are shown for the case (a) in which the observation is the square root of the state variable being updated and (b) in which the state variable is located two grid intervals away from the observed variable.

observation configuration is less relevant to atmospheric applications.

For linear, Gaussian problems, standard regression provides the best linear, unbiased estimate (Anderson 2003). However, for general nonlinear, non-Gaussian problems there is no form of regression that leads to a solution that is consistent with the optimal Bayesian solution. Rank regression is just one possible choice and empirical validation in the next section can be used to compare various choices.

The behavior of an ensemble filter with rank regression could be examined for specific cases for which the 
analytic solution is known, for instance cases in which the prior is drawn from a bivariate Gaussian with known parameters. The rank regression method does not converge to the correct solution as the ensemble size increases and has very complex behavior as ensemble size or prior correlation varies. An examination of this behavior is beyond the scope of this report.

\section{Results}

Initially, results for random observation configurations with 80 member ensembles are discussed for each of the different forward operators. Other ensemble sizes and uniform observation station configurations are examined subsequently.

\section{a. Identity forward operator}

Figure 2 shows the RMSE of the best treatment for each identity forward operator case with the random observation configuration. A standard regression treatment produces the lowest RMSE for all of these cases. In general, the difference in RMSE between the best rank regression treatment and the best standard regression treatment has high statistical significance (significance level $p$ value $<0.00001$ for all but three cases). For the cases with small RMSE, the best treatment generally uses an EnKF ( 7 of 20 cases), but for larger RMSE cases the RHF produces the lowest RMSE.

Figure 3 shows the lowest RMSE for each of the six treatments for cases with observation error variance of 4. For all five assimilation periods, the RMSE of the best standard regression treatment is a few percent smaller than that of the best rank regression. The differences are all highly significant; therefore, standard regression is clearly the treatment of choice. The observation space treatment is also important; the RHF has smaller RMSE for all standard regression cases.

Figure 4 displays the time mean of the ratio of posterior ensemble spread to RMSE for all 20 cases with identity forward operators and random observation configuration. This ratio is expected to have a time mean value of 1 for linear, Gaussian systems for which the Kalman filter solution is optimal. Figure 4a shows that the ratio varies between about 0.93 and 1.13 for rank regression treatments and is generally larger for smaller observation error variance cases. Figure $4 \mathrm{~b}$ shows that the ratio varies between about 0.9 and 1.08 for standard regression treatments. The ratio still tends to be larger for smaller observation error variance although the relationship is not as strong as for rank regression. Minimum RMSE is not necessarily found for treatments with spread to RMSE ratios of 1 , even in the relatively simple L96 system. When tuning an ensemble filter system in

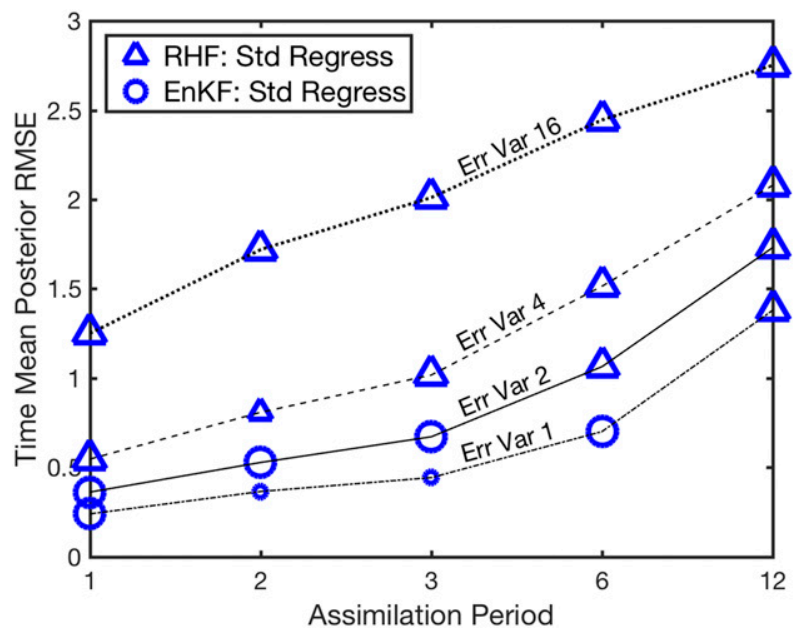

FIG. 2. Time mean posterior RMSE for the best assimilation method for each of the 20 cases with randomly located observation stations and identity forward operators. The four symbols for each assimilation period are for the four values of observation error variance. The standard regression gave the lowest time-averaged posterior RMSE for all cases, and therefore all symbols are blue. A triangle indicates that the lowest RMSE happened with a rank histogram filter in observation space, and a circle indicates that the EnKF was best. A large symbol indicates that the difference between the best rank regression case and the best standard regression case had a $p$ value of less than 0.00001 , an intermediate symbol indicates a $p$ value of less than 0.01 , and a small symbol is for a $p$ value of greater than 0.01 .

larger models, it is likely that trade-offs will be required between smaller RMSE and an ability to interpret the spread as an estimate of uncertainty; optimal RMSE may not be associated with good calibration of ensemble variance. These results suggest that this issue might be somewhat more important for rank regression than standard regression.

\section{b. Square root forward operator}

Figure 5 shows the RMSE of the best treatment for each of the cases for the square root forward operator. For cases with larger RMSE, the best treatments apply rank regression with an RHF. For smaller RMSE, the best treatments use standard regression with an EnKF. The differences between the best rank and standard treatments are highly significant for most of the cases in which the rank results are better; however, most of the cases in which the standard regression is better do not have statistically significant differences. In all of these cases, the median value of RMSE over the 10 experiments is smaller for rank regression with the RHF than for any of the standard regression treatments. However, for the cases with shorter observation period, a few of the rank regression experiments have much larger RMSE due to periods when the ensemble diverges from 


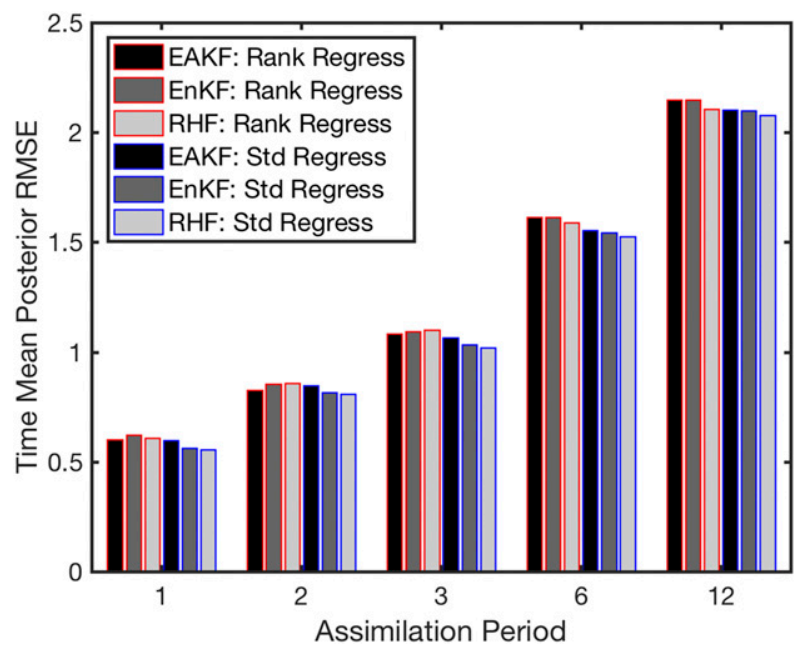

FIG. 3. Time mean posterior RMSE for the best assimilation for each of the six treatments as a function of assimilation period for randomly located observation stations with identity forward operators with observation error variance of 4.0. The six bars for each period are, from left to right, rank regression with EAKF, rank regression with EnKF, rank regression with RHF, standard regression with EAKF, standard regression with EnKF, and standard regression with RHF. Bars with red boundaries are for rank regression; bars with blue boundaries are for standard regression.

the truth. Such periods also occur for the standard regression, but less frequently and with shorter duration. It is possible that an adaptive inflation algorithm might reduce the frequency of divergence, but that has not been explored here.

Figure 6 shows RMSE for all six treatments for cases with observation error variance of 2 . Again, the differences between the best rank and standard regression treatments are normally a few percent although the difference is larger than $10 \%$ for observations every three time steps. Rank regression treatments without the RHF produce much larger RMSE than the other four treatments. Since the observation space RHF deals more effectively with non-Gaussian marginal distributions for observations than the EnKF or EAKF, this result suggests that the rank regression produces observation space priors that are significantly non-Gaussian giving the RHF a significant advantage.

Figure 7 displays the localization values for the best treatments for all 20 cases. The localization for the best rank treatments (Fig. 7a) is almost always tighter than for the corresponding standard regression treatments (Fig. 7b). The best localization generally gets tighter as the assimilation period gets longer or the observation error variance gets larger. In other words, tighter localization is required for cases with larger RMSE, however there are some exceptions. Rank regression cases with the smallest error variance $(0.25)$ and more frequent
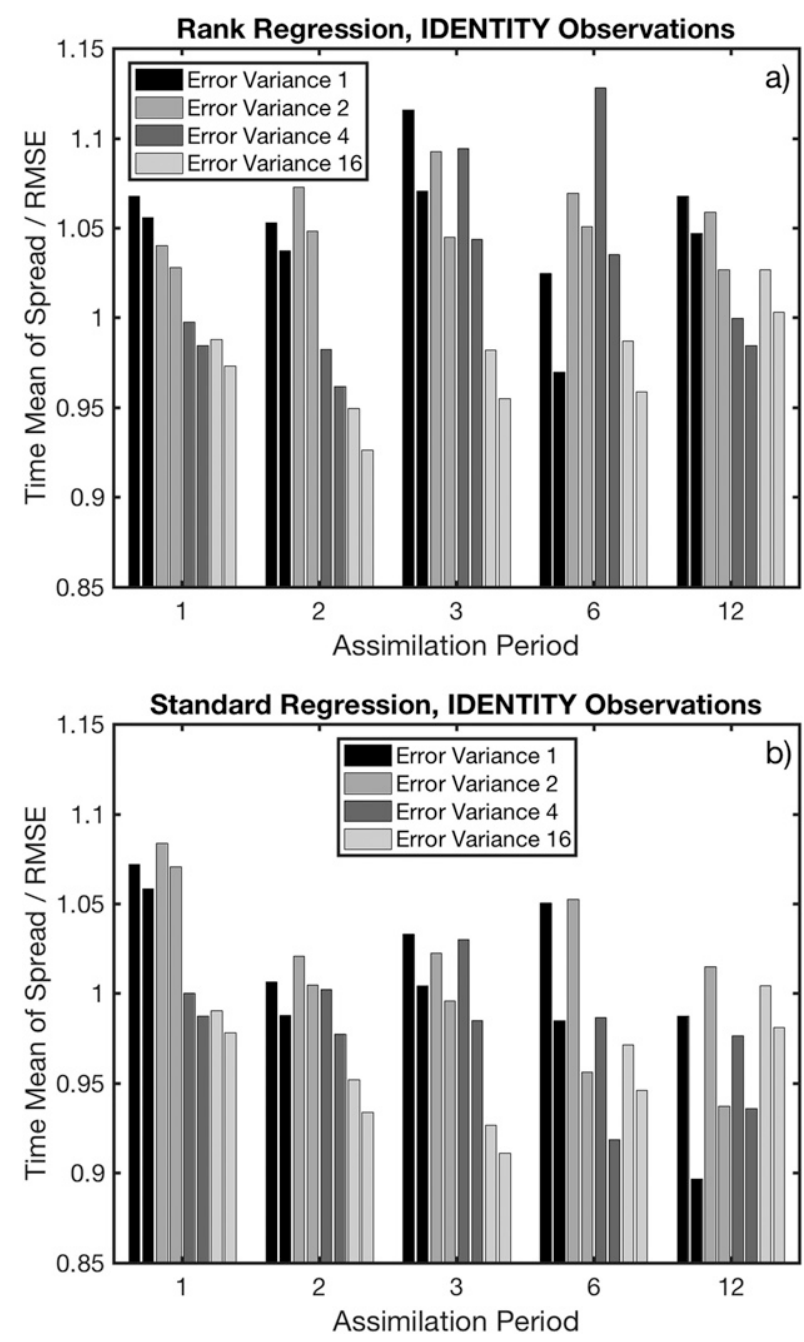

FIG. 4. For (a) rank regression and (b) standard regression, the time mean of the ratio of spread to ensemble mean RMSE for the assimilation with smallest time mean posterior RMSE for each of the four observation error variances as a function of assimilation period for randomly located observation stations with identity forward operators. Pairs of bars with the same shading are the prior and posterior ratios. The four pairs of bars for each assimilation period are for the four values of observation error variance from smallest on the left to largest on the right.

observations (every 1,2, or 3 time steps) have tighter localization than cases with error variance 0.5. Examination of the individual assimilation experiments shows that in cases with error variance 0.25 , the ensemble occasionally diverges from the true trajectory for short periods of time. Rank regression treatments with broader localization have smaller ensemble mean errors for most of the 5000 steps, but diverge more frequently.

Figure 8 shows the inflation values for the best treatments for all 20 cases. The best standard regression treatments (Fig. 8b), often have no inflation (1.0) and never have inflation larger than 1.05. Inflation is larger 


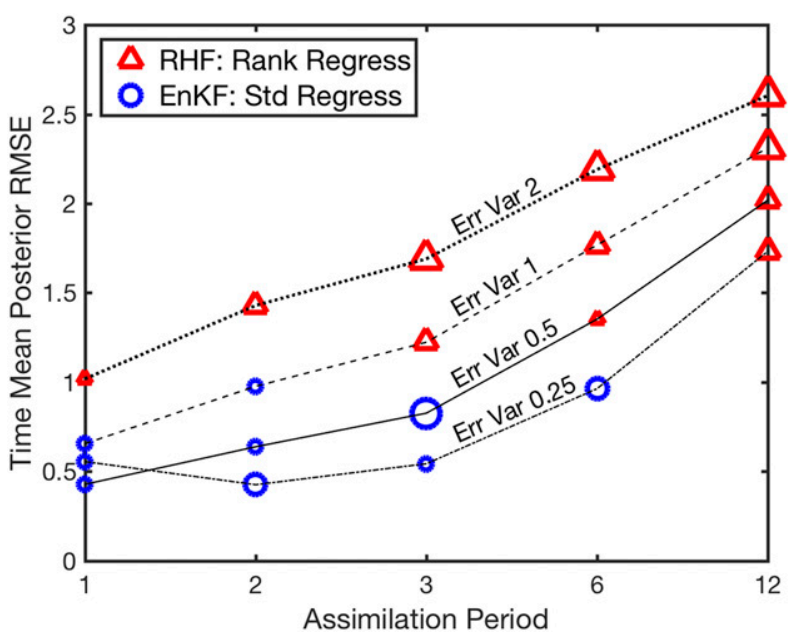

FIG. 5. Time mean posterior RMSE for the best assimilation method for each of the 20 cases with randomly located observation stations and square root forward operators. The four symbols for each assimilation period are for the four values of observation error variance. A red symbol indicates that rank regression had the smallest RMSE, and a blue symbol means standard regression was best. A triangle indicates that the lowest RMSE happened with a rank histogram filter in observation space, and a circle indicates that the EnKF was best. A large symbol indicates that the difference between the best rank regression case and the best standard regression case had a $p$ value of less than 0.00001 , an intermediate symbol indicates $p$ value of less than 0.01 , and a small symbol is for a $p$ value of greater than 0.01 .

for the best rank treatments with values for assimilation periods of 2,3, and 6 time steps that are greater than 1.05 for some observation error variances. These larger inflation values are apparently necessary to avoid periodic filter divergence that occurs in many of these cases with smaller inflation as noted above. An RHF often requires larger inflation than an EnKF or EAKF (Anderson 2010), so the larger inflation may be expected.

\section{c. Cube forward operator}

Figure 9 shows the RMSE of the best treatment for each of the cases for the cube forward operator. For cases with larger assimilation period, the best treatments use rank regression with an RHF. Smaller RMSE cases generally use standard regression and an EnKF. Several cases with intermediate RMSE use rank regression with an EnKF or standard regression with an RHF, but RMSE differences in these cases are not statistically significant.

Figure 10 shows the RMSE for all six treatments for cases with observation error variance of 1024 . The difference in RMSE between the best rank and standard regression treatments is more than $10 \%$ for all assimilation periods; rank regression is best for observations taken every 6 and 12 time steps, and standard regression

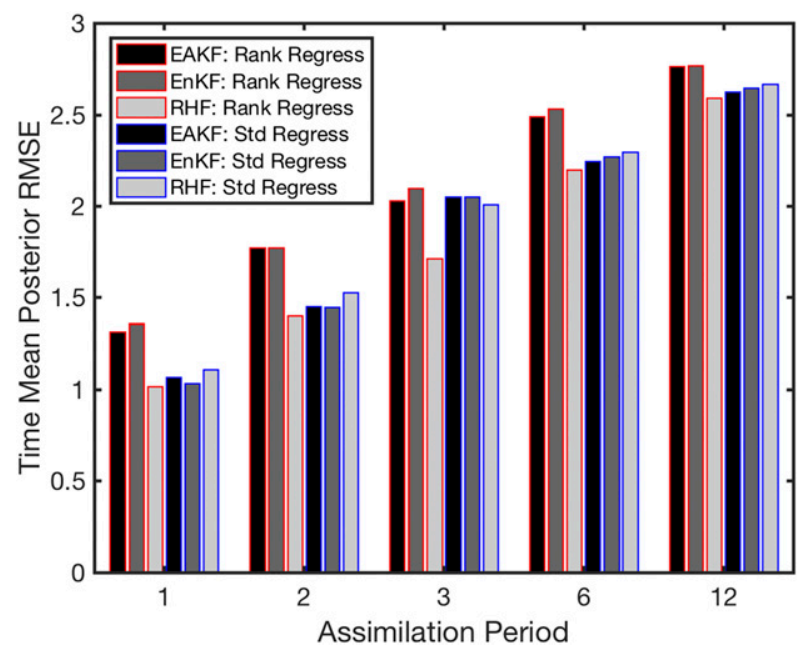

FIG. 6. Time mean posterior RMSE for each of the six treatments as a function of assimilation period for randomly located observation stations with square root forward operators with observation error variance of 2.0. The six bars for each period are, from left to right, rank regression with EAKF, rank regression with EnKF, rank regression with RHF, standard regression with EAKF, standard regression with EnKF, and standard regression with RHF.

is better for shorter assimilation periods. Despite the large difference between the best standard and rank treatments for observations every 1,2, and 3 steps, the differences are not statistically significant with only 10 experiments. This is because experiments are characterized by occasional filter divergence that is usually followed by a "reacquisition" of the true trajectory. In almost all cases in the entire study where the $p$ value is less than 0.00001 , all 10 experiments from the better regression have smaller RMSE than the corresponding experiment with the other regression. For the cube forward operator cases without significant differences, this is not true. The number of experiments for which standard regression has smaller RMSE than rank for these cases is between 4 and 7, but one regression may diverge much more frequently than the other. Again, these divergence issues might be reduced with the use of an adaptive inflation algorithm.

\section{d. Square forward operator}

Figure 11 shows the RMSE of the best treatment for each of the cases for the square forward operator. The function that maps state to observation no longer has an inverse in this case. The implicit assumption in the rank regression of a monotonic functional relation between the state and observation is violated in this case. The results show that standard regression with an RHF is most frequently the best treatment. There are also cases with standard regression and the EnKF for smaller 

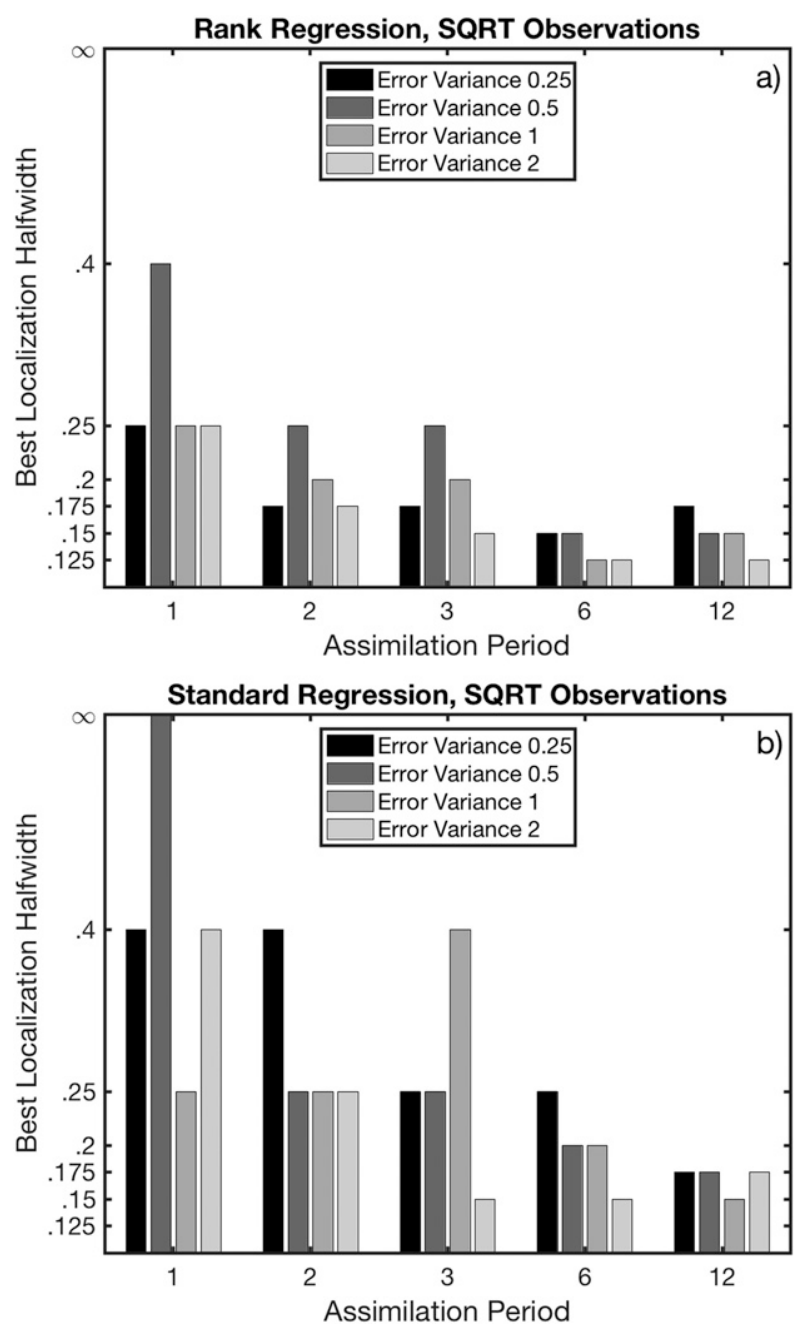

FIG. 7. For (a) rank regression and (b) standard regression, localization half-width with smallest time mean posterior RMSE for randomly located observation stations with square root forward operators. The four bars for each assimilation period correspond to the observation error variance, with the smallest variance on the left.

RMSE and a single instance where an EAKF with standard regression is best. The results suggest that rank regression is not generally effective for this forward operator. However, the performance of the standard regression is also probably poor in these cases relative to more general methods like particle filters (van Leeuwen 2009) or localized particle filters (Poterjoy 2016).

\section{e. Different ensemble sizes}

Ensemble sizes of 20, 40, and 160 were also used with the random observation station configuration cases with identity and square root forward operators. For identity forward operators, standard regression still produced the lowest RMSE for all cases for all ensemble sizes. However, for 20 member ensembles, the EAKF had the
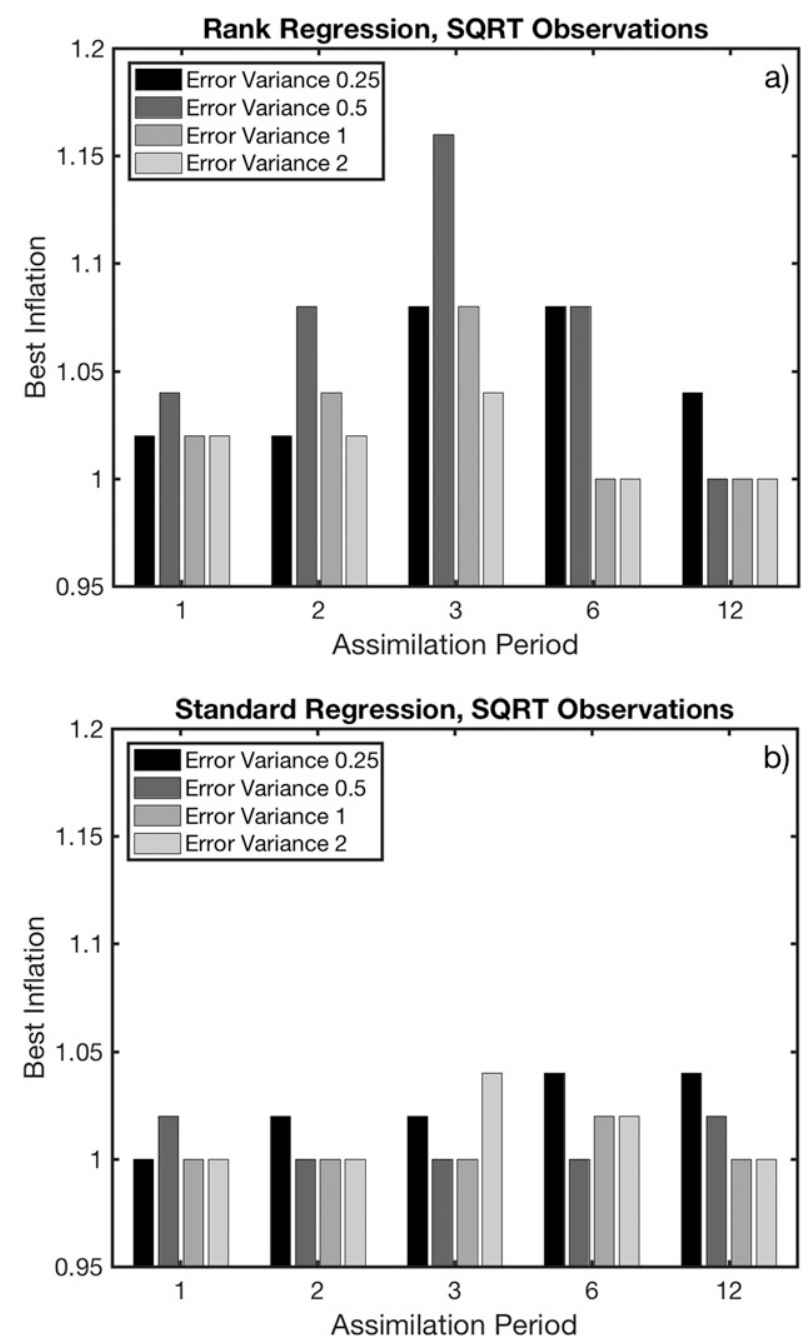

FIG. 8. Similar to Fig. 7, but for inflation with smallest time mean posterior RMSE for randomly located observation stations with square root forward operators.

lowest RMSE for all but one pair of observation error variance and assimilation period. For 40 members, the EAKF, EnKF and RHF each produced the lowest RMSE for several pairs of observation error variance and assimilation period. For 160 members, the RHF produced lowest RMSE for all but four pairs of observation error variance and assimilation period, the ones for the smallest observational error variance.

Results for the square root forward operator showed more variability as a function of ensemble size. With 20 ensemble members, standard regression with the EAKF produced the smallest RMSE for all cases. For 40 ensemble members, Fig. 12 shows results are more similar to the 80 member results. The best treatments for large RMSE use rank regression with an RHF. For the smallest RMSE cases, the best treatments mostly use standard regression with an EnKF while for several 


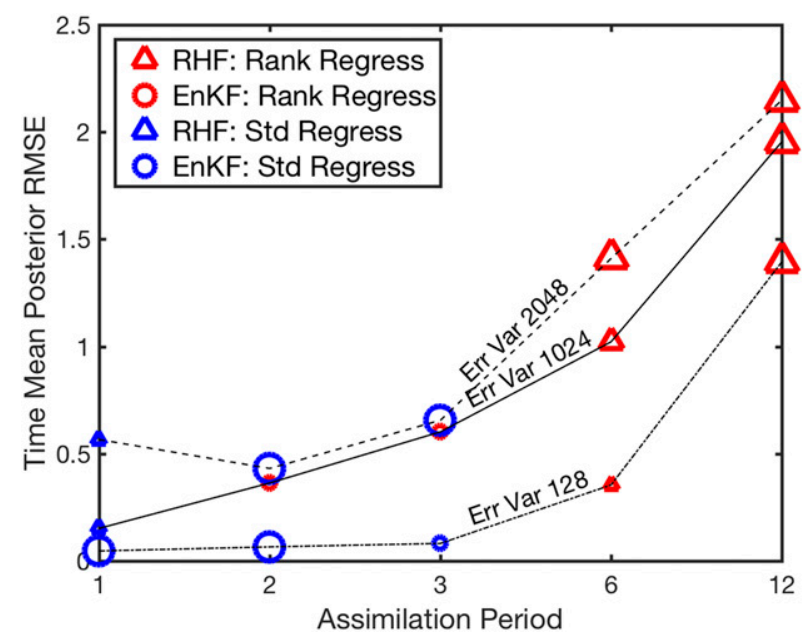

FIG. 9. Time mean posterior RMSE for the best assimilation treatment for each of the 20 cases with randomly located observation stations and cube forward operators. The three symbols for each assimilation period are for the three values of observation error variance. A red symbol indicates that rank regression had the smallest RMSE, and a blue symbol means standard regression was best. A triangle indicates that the lowest RMSE happened with a rank histogram filter in observation space, and a circle indicates that the EnKF was best. A large symbol indicates that the difference between the best rank regression case and the best standard regression case had a $p$ value of less than 0.00001 , an intermediate symbol indicates $p$ value of less than 0.01 , and a small symbol is for a $p$ value of greater than 0.01 .

intermediate RMSE cases, the best treatment uses standard regression with the EAKF. For 160 members, Fig. 13 shows that results are similar to the 80 member results in Fig. 5. There are fewer cases for which the RMSE difference has the highest level of statistical significance. These results suggest that rank regression is not effective in small ensembles, but has consistent advantages for nonlinear forward operators with larger ensembles and larger RMSE. If the RMSE is small, the prior ensemble bivariate distributions of an observation and a state variable may still appear quite linear because of the limited range of uncertainty. In these cases, there is little to be gained by attempting to more accurately represent the nonlinear relation that may exist.

Figure 14 summarizes the RMSE as a function of ensemble size for the square root forward operator with assimilation period 3. In all cases, the RMSE decreases as a function of ensemble size for each observation error variance. For the smallest error variance case, 0.25 , rank regression has much larger errors than standard regression and improves little with increasing ensemble size. For larger error variance, and hence larger RMSE, the improvement with ensemble size for rank regression is much larger. For observation error variance 1 and 2, the rank regression RMSE for 80

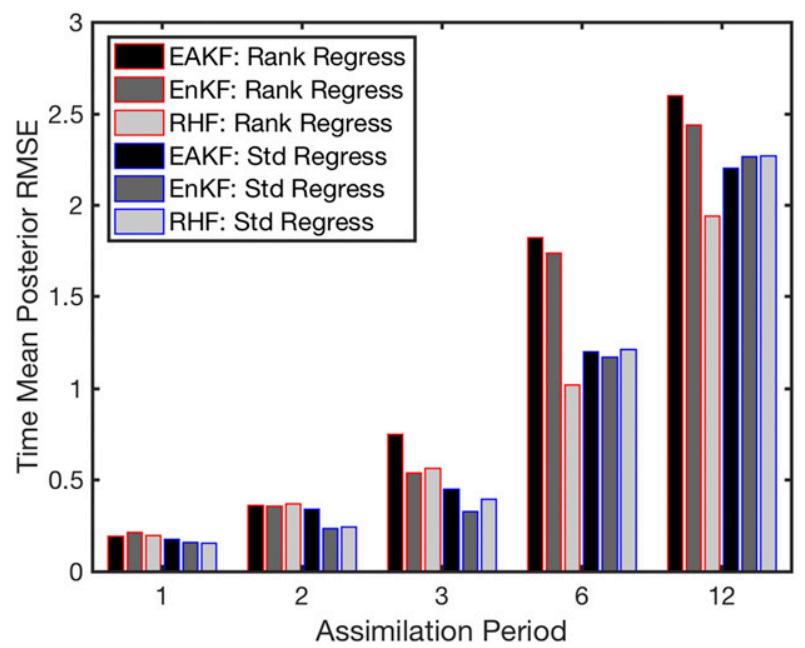

FIG. 10. Time mean posterior RMSE for each of the six treatments as a function of assimilation period for randomly located observation stations with cube forward operators with observation error variance of 1024. The six bars for each period are, from left to right, rank regression with EAKF, rank regression with EnKF, rank regression with RHF, standard regression with EAKF, standard regression with EnKF, and standard regression with RHF.

members is smaller than the 160 member RMSE for standard regression.

\section{f. Uniform observation station configurations}

Results were also produced with 80 ensemble members for uniform observation station configurations for identity, square root, and cube forward operators. In these cases, each observation impacts one state variable where the prior ensemble is exactly on the continuous curve corresponding to the forward operator. For example, Fig. 1a shows the prior ensemble from a case with the square root forward operator. Rank regression that respects this prior relation might have a distinct advantage over standard regression in this situation. However, each observation also impacts up to 39 other state variables (depending on the localization) for which the prior ensemble is more similar to the example in Fig. 1b. Results for uniform observation station configurations were generally similar to those for random station configurations. For the identity forward operator, standard regression was always better than rank regression with the RHF being used in the best treatment for larger RMSE and the EnKF for smaller RMSE. For nonlinear forward operators, rank regression with the RHF was best for larger RMSE while standard regression was better for smaller RMSE. However, the rank regression was better for smaller values of RMSE with the uniform configurations than for random configurations. This is consistent with rank regression being especially relevant for the situation in Fig. 1a. 


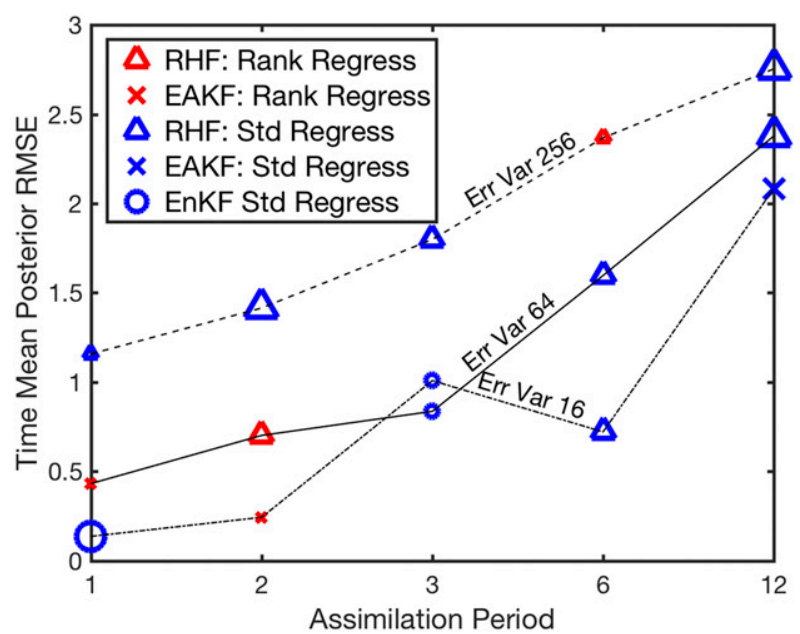

FIG. 11. Time mean posterior RMSE for the best assimilation treatment for each of the 20 cases with randomly located observation stations and square forward operators. The three symbols for each assimilation period are for the three values of observation error variance. A red symbol indicates that rank regression had the smallest RMSE, and a blue symbol means standard regression was best. A triangle indicates that the lowest RMSE happened with a rank histogram filter in observation space, a circle indicates that the EnKF was best, and an $\mathrm{X}$ indicates that the EAKF was best. A large symbol indicates that the difference between the best rank regression case and the best standard regression case had a $p$ value of less than 0.00001 , an intermediate symbol indicates $p$ value of less than 0.01 , and a small symbol is for a $p$ value of greater than 0.01 .

\section{Computational scaling and cost}

Rank regression is considerably more complicated to compute than standard regression. Here, the discussion of cost and scaling is for the regression step for a single observation, state variable pair. For the standard regression, the cost is $O(N)$, where $N$ is the ensemble size and involves the computation of the sample covariance matrix.

Two additional computationally intensive steps are required to compute the rank regression. First, the prior state and observation ensembles must be sorted in order to compute ranks. Sorting algorithms that are $O(N \log N)$ on average and in the worst case are available. Second, given a prior ensemble, it is necessary to compute the continuous ranks for each element of a vector of $N$ values. For each of the $N$ values, this requires a search in a sorted vector of $N$ values. Using a binary search, the average and worst case are $O(\log N)$, and therefore the cost for $N$ searches is also $O(N \log N)$.

However, it is possible to reduce the computational scaling in practice. Each state variable is updated once by each observation at which point the state ensemble must be sorted. For the application here, and more generally for large geophysical applications, a single observation usually does not randomly scramble the

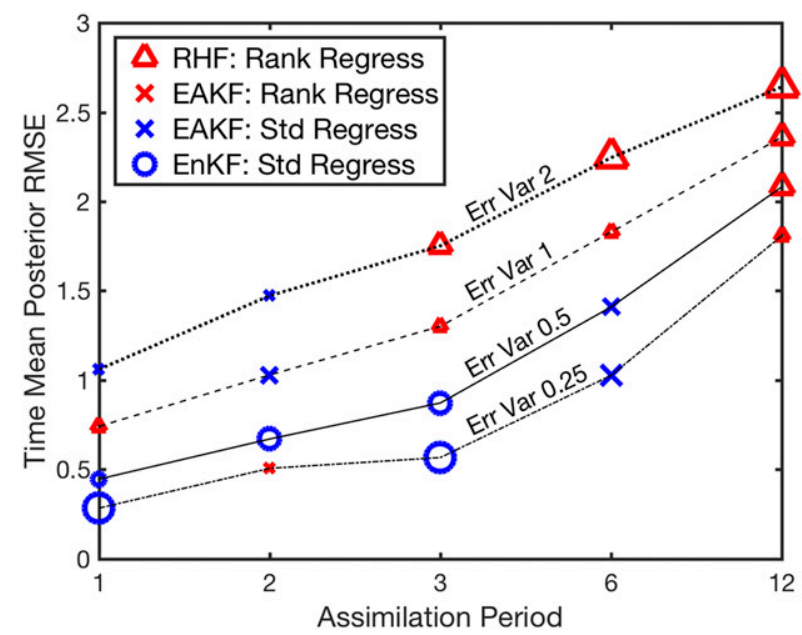

FIG. 12. Time mean posterior RMSE for the best assimilation method for each of the 40 cases with randomly located observation stations and square root forward operators. The four symbols for each assimilation period are for the four values of observation error variance. A red symbol indicates that rank regression had the smallest RMSE, and a blue symbol means standard regression was best. A triangle indicates that the lowest RMSE happened with a rank histogram filter in observation space, a circle indicates that the EnKF was best, and an X indicates that the EAKF was best. A large symbol indicates that the difference between the best rank regression case and the best standard regression case had a $p$ value of less than 0.00001 , an intermediate symbol indicates $p$ value of less than 0.01 , and a small symbol is for a $p$ value of greater than 0.01 .

state ensemble. Instead, an observation tends to make minor modifications so that the posterior ensemble has nearly the same rank statistics as the prior. In this case, it is possible to apply sorting algorithms that have better scaling for sorting arrays of values that are already nearly sorted. A simple insertion sort can produce $O(N)$ scaling for nearly sorted arrays. Examination of scaling results for sample cases with ensemble sizes ranging from 20 to 5120 (increasing size by factors of 2) indicates that the scaling for using an $O(N \log N)$ algorithm such as "quicksort" is as expected, but insertion sort results in $O(N)$ scaling.

A similar approach can be taken for the conversion of state values to continuous ranks given an ensemble. The values being converted are the posterior values corresponding to the prior ensemble. Again, it is normally the case that the rank of the posterior does not change drastically from its prior rank after assimilating a single observation. A linear search starting in the vicinity of the prior rank can be faster than a binary search in this case. Again, tests covering the large range of ensemble sizes indicates that the scaling is $O(N)$ for the linear search.

While this scaling is encouraging, the actual cost for the Lorenz96 cases here is significantly larger for the rank regression than for the simple regression. For just 


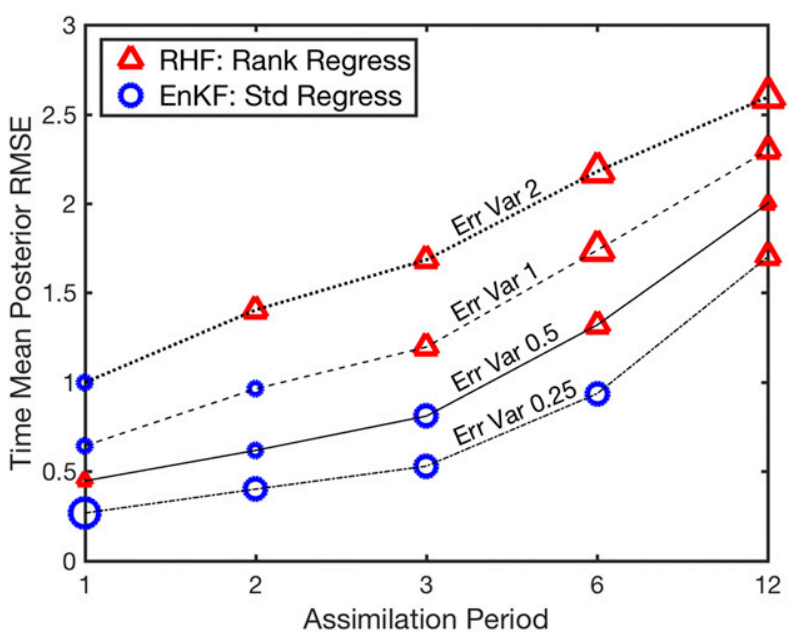

FIG. 13. Time mean posterior RMSE for the best assimilation method for each of the 160 cases with randomly located observation stations and square root forward operators. The four symbols for each assimilation period are for the four values of observation error variance. A red symbol indicates that rank regression had the smallest RMSE, and a blue symbol means standard regression was best. A triangle indicates that the lowest RMSE happened with a rank histogram filter in observation space, and a circle indicates that the EnKF was best. A large symbol indicates that the difference between the best rank regression case and the best standard regression case had a $p$ value of less than 0.00001 , an intermediate symbol indicates $p$ value of less than 0.01 , and a small symbol is for a $p$ value of greater than 0.01 .

the regression part of the algorithm, the ratios of computational cost on a single processor are approximately 5, 8, 10, and 12 for ensemble sizes of 20, 40, 80, and 160 . The regression is just a fraction of the total cost, so the ratios for the total filtering time are smaller and vary widely depending on the localization and assimilation period. For long assimilation periods with small localization, costs for the total solution can be roughly comparable.

For large geophysical applications, the relative cost of the model advance to the filtering can be much larger than for these Lorenz96 cases. Of course, this depends on details like the number of observations, the number of state variables, and the localization. Testing will be required to determine the relative cost of rank regression for large applications. In order for rank regression to be of interest, the RMSE for rank regression must be less than the RMSE for an equally costly standard regression. This requires rank regression with ensemble size $N$ to produce smaller RMSE than standard regression with an ensemble size greater than $N$. Results in Fig. 14 show that this occurs in low-order applications. Further exploration is required to see whether it is the case for some large applications.

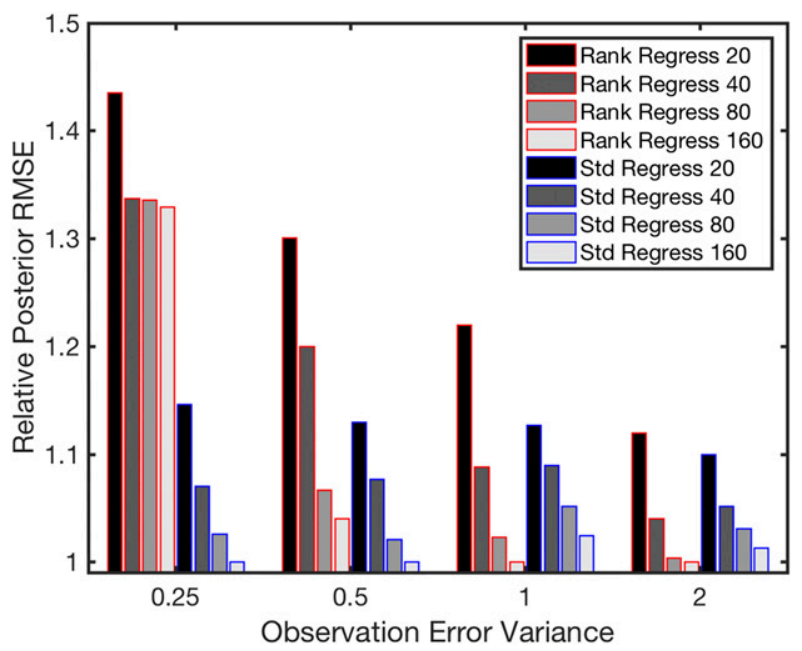

FIG. 14. Ratio of the time mean posterior RMSE to the best RMSE with ensemble size 160 for square root observations with assimilation period 3 . The first four bars for each observation error variance are for rank regression with ensemble sizes of 20,40, 80, and 160; the second four bars are for standard regression. Bars with the same fill color are for the same ensemble size.

\section{Discussion and conclusions}

A method for nonlinear regression in an ensemble filter has been described and tested in a low-order model. Rank regression produces smaller posterior RMSE than standard linear regression in some cases with nonlinear forward operators. One could also apply more specific forms of nonlinear regression, for instance polynomial regressions, that were tailored to the details of a specific forward operator. Because it implicitly assumes a monotonic functional relation between the state variable and observation, rank regression is less effective for forward operators that are not invertible like the square forward operator. More general filtering methods like particle filters are required for good performance in such cases.

Many other filtering methods that work well with nonlinear problems have been developed, for instance the maximum likelihood ensemble Filter (Zupanski 2005). Another example, the iterative EnKF (IEnKF) that is described in Sakov et al. (2018), is likely to perform better than any regression-based method for strongly nonlinear problems, exactly the ones for which the rank regression has advantages over standard regression. Comparison of both the quality and cost of the IEnKF and regressionbased methods would be an interesting area for further research.

Simon and Bertino (2009) applied a Gaussian anamorphosis method designed to make prior distributions more Gaussian via a univariate mapping of the prior. This leads to improved assimilation results in 
applications with non-Gaussian priors. One could envision doing similar remappings to make the prior relation between the state and observation more linear, as opposed to making one of the prior marginals more Gaussian.

This study also explored the performance of three different updates in observation space. The choice of observation space update often had significant impacts on the RMSE. The best observation update depended on details of the assimilation problem including the ensemble size, the assimilation period, the observation error variance, and the type of regression employed. The non-Gaussian rank histogram filter was usually essential for good performance with rank regression, suggesting that this regression led to less-Gaussian priors in observation space. The RHF also was the best choice for many problems with larger ensemble size with standard regression. The perturbed observation ensemble Kalman filter was the best choice for larger ensembles in cases with relatively small RMSE. The deterministic ensemble adjustment Kalman filter was best for many cases with small ensemble size. These results have been reported previously, but this study makes a comprehensive examination over a large number of cases.

A common theme from many comparative studies of nonlinear and non-Gaussian ensemble filter methods is that conditional application of more advanced methods is worth exploring. For instance, one could determine a priori, or try to detect on the fly, cases in which priors are particularly nonlinear or non-Gaussian and apply an appropriate method in these cases. More advanced algorithms tend to be more expensive, so conditional application may result in improved performance without excessive cost.

Even small details of standard ensemble algorithm implementations can lead to large changes in RMSE. For example, the EnKF algorithm used here sorts the posterior in observation space (Anderson 2003) so that the average value of the observation increments is minimized. This does not change the answer in linear Gaussian cases, but by minimizing the average increment in state space, it minimizes errors in cases in which the prior distributions are nonlinear. All cases for identity forward operators with random observation station configuration were repeated with an EnKF without sorting. Figure 15 shows the ratio of posterior RMSE for the best inflation/localization pair without sorting to the best case with sorting. For all cases sorting leads to smaller RMSE with a reduction of several percent in most cases. Getting good performance from an EnKF requires this sorting, which may be difficult to implement in filters that do not serially assimilate observations.

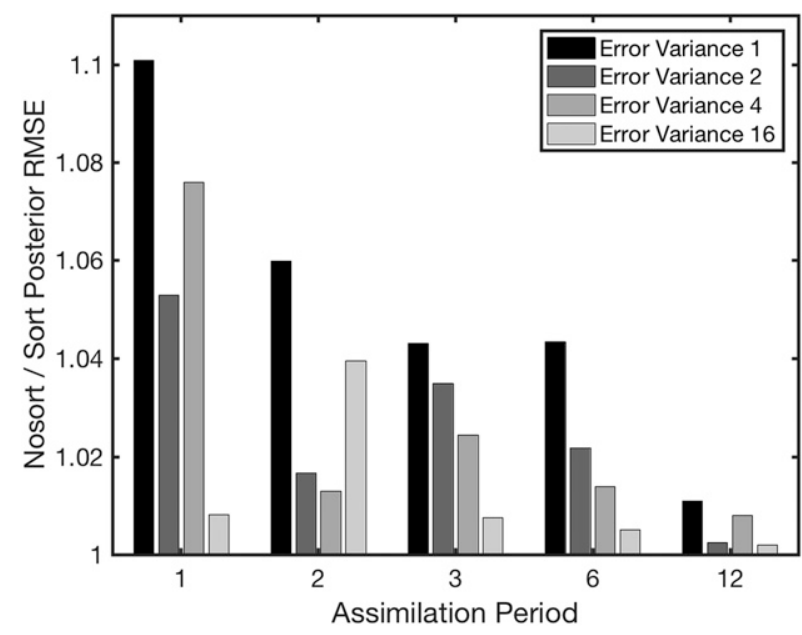

FIG. 15. Ratio of posterior RMSE from EnKF without sorting of observation increments to EnKF with sorting of observation increments for standard regression and identity forward operators with uniform observing station configuration, produced with 80 ensemble members. The four bars for each assimilation period correspond to the four observation error variances, with the smallest variance on the left.

Another detail that was not explored in this study is the impact of parallel filter algorithms. The standard DART parallel algorithm (Anderson and Collins 2007) that computes forward operators for all observations at a given time at once before assimilating any of them was used here. An algorithm in which the first observation is assimilated before computing the next forward operator could significantly impact RMSE but would also impact the scalability.

Significant impacts on RMSE can be obtained by modifying ensemble filter algorithms in both observation space and for regression. Careful tuning, or good adaptive algorithms, for inflation and localization are also essential for good performance and values may vary widely for different observing systems. Low-order model results here suggest that there is lots of room to improve ensemble filter performance for larger applications. In particular, careful exploration of ensemble filter methods and tuning might lead to a reduction of RMSE for numerical weather prediction applications by many percent, improvements that are highly significant for these systems.

Acknowledgments. The author is grateful for support from the DART team, Nancy Collins, Moha El Gharamti, Johnny Hendricks, Tim Hoar, and Kevin Raeder. Stephen Anderson helped to improve and verify the presentation of the method. Thanks are given to three anonymous reviewers for comments that led to a much better presentation of the results. The National 
Center for Atmospheric Research is sponsored by the National Science Foundation. Any opinions, findings, and conclusions or recommendations expressed in this publication are those of the author and do not necessarily reflect the views of the National Science Foundation.

\section{APPENDIX}

\section{Mapping Values to Ranks and Ranks to Values}

The rank regression method requires an invertible function:

$\tilde{r}=f(\tilde{x} ; x) \quad$ and $\quad \tilde{x}=f^{-1}(\tilde{r} ; x), \quad \tilde{x}=f^{-1}(\tilde{r} ; x)$,

where $x=\left(x_{1}, x_{2}, \ldots, x_{N}\right)$ is an ensemble, $\tilde{x}$ is a value, and $\tilde{r}$ is a continuous generalized rank, a real number. Let $\mathbf{s}=\left(s_{1}, s_{2}, \ldots, s_{N}\right)$ be the vector of the sorted values of $\mathbf{x}$ so that $s_{1}$ is the smallest value in $\mathbf{x}, s_{2}$ is the second smallest, and so on. The function $f$ is defined as

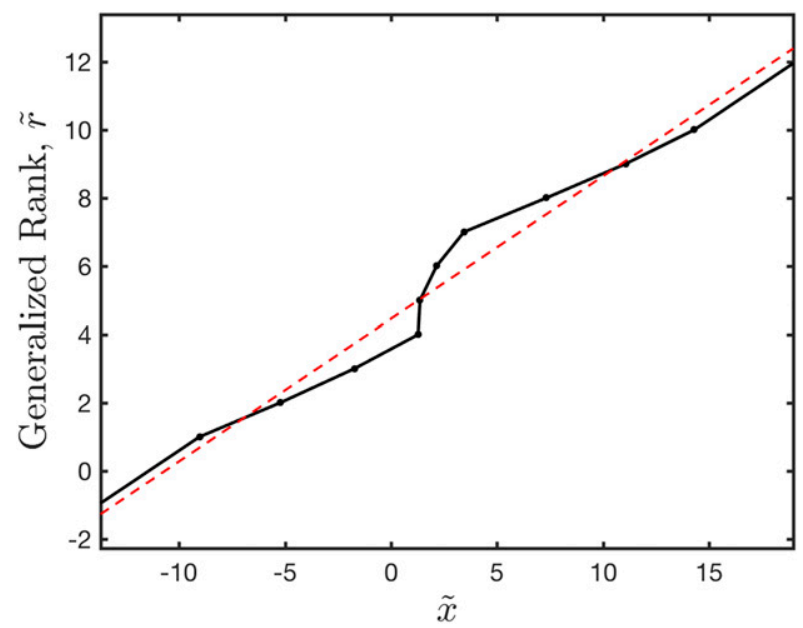

FIG. A1. An example of the function $f$ (black) from values $\tilde{x}$ to continuous generalized rank $\tilde{r}$ given a 10 -member ensemble randomly drawn from a normal distribution. The ensemble values vs their rank statistics are indicated by the black dots. The red dashed line is the least squares fit to the black dots whose slope is used to extrapolate the function beyond the smallest and largest values of the ensemble.

$$
f(\tilde{x} ; x)= \begin{cases}1-b\left(s_{1}-\tilde{x}\right), & \tilde{x}<s_{1} \\ n+\left(\tilde{x}-s_{n}\right) /\left(s_{n+1}-s_{n}\right), & s_{n} \leq \tilde{x} \leq s_{n+1} . \\ N+b\left(\tilde{x}-s_{N}\right), & \tilde{x}>s_{N}\end{cases}
$$

This is a linear interpolation in rank when $\tilde{x}$ is between the smallest and largest values of $\mathbf{x}$. An extrapolation is required if $\tilde{x}$ is smaller than the smallest value in $\mathbf{x}$ or larger than the largest value. Define $r_{n}=n, n=1,2, \ldots$, $N$ and let $b$ be the slope of the least squares fit line for $\mathbf{r}$ as a function of $\mathbf{s}$, that is, the value of $b$ that minimizes

$$
\sum_{n=1}^{N}\left[r_{n}-\left(a+b s_{n}\right)\right]^{2}
$$

Figure A1 shows a sample of the function $f$ for a small ensemble randomly drawn from a normal distribution.

The details of the extrapolation can have a large impact on the results when using rank regression. The impact could be even greater in cases in which the observations are biased compared to the model prior as this would lead to a larger fraction of updated ranks being outside the original range from 1 to $N$. The extrapolation as implemented here implicitly assumes a linear relation between the sorted ensemble and the corresponding ranks, but this may be a poor choice for some prior ensemble distributions. It is possible to significantly improve the performance of the rank regression by changing to other types of extrapolation and this deserves further study.
The experiments reported here computed nearly 250 billion regressions, and there was never an instance in which an ensemble $\mathbf{x}$ contained duplicate values to machine precision. However, for other types of models this situation could occur frequently. In that case, $f$ would have to be extended to handle cases in which $s_{n}=s_{n+1}$.

\section{REFERENCES}

Anderson, J. L., 1996: A method for producing and evaluating probabilistic forecasts from ensemble model integrations. J. Climate, 9, 1518-1530, https://doi.org/10.1175/1520-0442(1996) 009<1518:AMFPAE $>2.0$.CO;2.

, 2001: An ensemble adjustment Kalman filter for data assimilation. Mon. Wea. Rev., 129, 2884-2903, https://doi.org/ 10.1175/1520-0493(2001)129<2884:AEAKFF>2.0.CO;2.

, 2003: A local least squares framework for ensemble filtering. Mon. Wea. Rev., 131, 634-642, https://doi.org/10.1175/15200493(2003)131<0634:ALLSFF>2.0.CO;2.

2009: Spatially and temporally varying adaptive covariance inflation for ensemble filters. Tellus, 61A, 72-83, https:// doi.org/10.1111/j.1600-0870.2008.00361.x.

_ 2010: A non-Gaussian ensemble filter update for data assimilation. Mon. Wea. Rev., 138, 4186-4198, https://doi.org/ 10.1175/2010MWR3253.1.

, 2016: Reducing correlation sampling error in ensemble Kalman filter data assimilation. Mon. Wea. Rev., 144, 913-925, https://doi.org/10.1175/MWR-D-15-0052.1. 
, and N. Collins, 2007: Scalable implementations of ensemble filter algorithms for data assimilation. J. Atmos. Oceanic Technol., 24, 1452-1463, https://doi.org/10.1175/JTECH2049.1.

, T. Hoar, K. Raeder, H. Liu, N. Collins, R. Torn, and A. Arellano, 2009: The Data Assimilation Research Testbed. Bull. Amer. Meteor. Soc., 90, 1283-1296, https://doi.org/ 10.1175/2009BAMS2618.1.

Bishop, C. H., 2016: The GIGG-EnKF: Ensemble Kalman filtering for highly skewed non-negative uncertainty distributions. Quart. J. Roy. Meteor. Soc., 142, 1395-1412, https://doi.org/ 10.1002/qj.2742.

Burgers, G., P. J. van Leeuwen, and G. Evensen, 1998: Analysis scheme in the ensemble Kalman filter. Mon. Wea. Rev., 126, 1719-1724, https://doi.org/10.1175/1520-0493(1998)126<1719: ASITEK $>2.0 . C O ; 2$.

El Gharamti, M., 2018: Enhanced adaptive inflation algorithm for ensemble filters. Mon. Wea. Rev., 146, 623-640, https://doi.org/ 10.1175/MWR-D-17-0187.1.

Gaspari, G., and S. E. Cohn, 1999: Construction of correlation functions in two and three dimensions. Quart. J. Roy. Meteor. Soc., 125, 723-757, https://doi.org/10.1002/qj.49712555417.

Hamill, T. M., 2001: Interpretation of rank histograms for verifying ensemble forecasts. Mon. Wea. Rev., 129, 550-560, https:// doi.org/10.1175/1520-0493(2001)129<0550:IORHFV> 2.0. CO;2.

Hodyss, D., 2012: Accounting for skewness in ensemble data assimilation. Mon. Wea. Rev., 140, 2346-2358, https://doi.org/ 10.1175/MWR-D-11-00198.1.

, J. L. Anderson, N. Collins, W. F. Campbell, and P. A. Reinecke, 2017: Quadratic polynomial regression using serial observation processing: Implementation within DART. Mon. Wea. Rev., 145, 4467-4479, https://doi.org/10.1175/MWR-D17-0089.1.

Houtekamer, P. L., and H. L. Mitchell, 1998: Data assimilation using an ensemble Kalman filter technique. Mon. Wea. Rev., 126, 796-811, https://doi.org/10.1175/1520-0493(1998) 126<0796:DAUAEK > 2.0.CO;2.

Lorenz, E. N., and K. A. Emanuel, 1998: Optimal sites for supplementary weather observations: Simulation with a small model. J. Atmos. Sci., 55, 399-414, https://doi.org/10.1175/ 1520-0469(1998)055<0399:OSFSWO >2.0.CO;2.
Posselt, D. J., and C. H. Bishop, 2018: Nonlinear data assimilation for clouds and precipitation using a gamma inverse-gamma ensemble filter. Quart. J. Roy. Meteor. Soc., 144, 2331-2349, https://doi.org/10.1002/qj.3374.

Poterjoy, J., 2016: A localized particle filter for high-dimensional nonlinear systems. Mon. Wea. Rev., 144, 59-76, https://doi.org/ 10.1175/MWR-D-15-0163.1.

Sakov, P., D. S. Oliver, and L. Bertino, 2012: An iterative EnKF for strongly nonlinear systems. Mon. Wea. Rev., 140, 1988-2004, https://doi.org/10.1175/MWR-D-11-00176.1.

_ J.-M. Haussaire, and M. Bocquet, 2018: An iterative ensemble Kalman filter in presence of additive model error. Quart. J. Roy. Meteor. Soc., 144, 1297-1309, https://doi.org/10.1002/qj.3213.

Simon, E., and L. Bertino, 2009: Application of the Gaussian anamorphosis to assimilation in a 3-D coupled physicalecosystem model of the North Atlantic with the EnKF: A twin experiment. Ocean Sci., 5, 495-510, https://doi.org/ 10.5194/os-5-495-2009.

Tippett, M. K., J. L. Anderson, C. H. Bishop, T. M. Hamill, and J. S. Whitaker, 2003: Ensemble square root filters. Mon. Wea. Rev., 131, 1485-1490, https://doi.org/10.1175/1520-0493(2003)131<1485: $\mathrm{ESRF}>2.0 . \mathrm{CO} ; 2$

van Leeuwen, P. J., 2009: Particle filtering in geophysical systems. Mon. Wea. Rev., 137, 4089-4114, https://doi.org/10.1175/ 2009MWR2835.1.

Vetra-Carvalho, S., P. Jan van Leeuwen, L. Nerger, A. Barth, J. U. Altaf, P. Brasseur, P. Kirchgessner, and J.-M. Beckers, 2018: State-of-the-art stochastic data assimilation methods for high-dimensional non-Gaussian problems. Tellus, $\mathbf{7 0 A}, 1-43$, https://doi.org/10.1080/16000870.2018.1445364.

Whitaker, J. S., and T. M. Hamill, 2012: Evaluating methods to account for system errors in ensemble data assimilation. Mon. Wea. Rev., 140, 3078-3089, https://doi.org/10.1175/MWR-D11-00276.1.

Zhang, F., C. Snyder, and J. Sun, 2004: Impacts of initial estimate and observation availability on convective-scale data assimilation with an ensemble Kalman filter. Mon. Wea. Rev., 132, 1238-1253, https://doi.org/10.1175/1520-0493(2004)132<1238: IOIEAO $>2.0 . \mathrm{CO} ; 2$

Zupanski, M., 2005: Maximum likelihood ensemble filter: Theoretical aspects. Mon. Wea. Rev., 133, 1710-1726, https:// doi.org/10.1175/MWR2946.1. 